Relations industrielles

Industrial Relations

\title{
Addresses on Industrial Relations (1957 Series). Bureau of Industrial Relations, University of Michigan, Ann Arbor, Bulletin No. 25. \$3.50.
}

\section{Louis-Marie Tremblay}

Volume 13, numéro 1, janvier 1958

URI : https://id.erudit.org/iderudit/1022486ar

DOI : https://doi.org/10.7202/1022486ar

Aller au sommaire du numéro

\section{Éditeur(s)}

Département des relations industrielles de l’Université Laval

\section{ISSN}

0034-379X (imprimé)

1703-8138 (numérique)

Découvrir la revue

Citer ce compte rendu

Tremblay, L.-M. (1958). Compte rendu de [Addresses on Industrial Relations (1957 Series). Bureau of Industrial Relations, University of Michigan, Ann Arbor, Bulletin No. 25. \$3.50.] Relations industrielles / Industrial Relations, 13(1), 117-117. https://doi.org/10.7202/1022486ar

Tous droits réservés @ Département des relations industrielles de l’Université Laval, 1958
Ce document est protégé par la loi sur le droit d'auteur. L'utilisation des services d'Érudit (y compris la reproduction) est assujettie à sa politique d'utilisation que vous pouvez consulter en ligne.

https://apropos.erudit.org/fr/usagers/politique-dutilisation/ 
ces des unions sur le niveau général des salaires.

Ce livre ne manquera pas d'intéresser un grand nombre de nos lecteurs pour de multiples raisons: les problèmes qui y sont discutés sont des problèmes contemporains: le plan des discussions est simple et logique; l'enchaînement des chapitres est aisé; l'absence d'appareils techniques d'analyse ne rebutera pas les non-initiés; la discussion, honnête et sérieuse, n'est jamais trop abstraite: des références statistiques nous raccrochent continuellement à la réalité, et les références biblographiques sont peu nombreuses et judicieusement choisies.

\section{J. St-L.}

\section{Addresses on Industrial Relations (1957} Series). Bureau of Industrial Relations, University of Michigan, Ann Arbor, Bulletin No. 25. $\$ 3.50$.

Dans cette publication sont groupées quatorze conférences données par des professeurs d'administration et des directeurs de personnel de grandes entreprises américaines. Les travaux furent présentés sous la direction du Département des Relations Industrielles de l'Université du Michigan au cours de réunions d'administrateurs dans quatre centres industriels du Michigan pendant l'année 1956-57. Le choix des sujets avait été préalablement déterminé par la décision majoritaire des participants qui délimitaient alors leurs besoins les plus urgents.

Ce groupe de chefs d'entreprise démontre un intérêt marqué pour les questions de formulation des politiques, de motivation des employés, d'amélioration des communications par la sélection du personnel de maîtrise. Cette insistance illustre la tendance actuelle qui vise à accroitre l'efficacité par une plus grande compétence. La première conférence donne le ton à la pensée commune qui anime tous les travaux. Les tendances nouvelles dans les relations humaines amènent la direction à repenser sa politique d'administration du personnel. La clé du problème, selon C.E. French, serait de développer chez l'individu un ensemble d'attitudes susceptibles de l'intégrer dans l'industrie, une volonté de produire, un sentiment d'appartenance à l'entreprise et la confiance en la direction. Pour celà, le département du personnel devra à l'avenir donner plus d'importance aux relations individuelles, effectuer plus de recherches, planifier davantage en longue période et exercer un contrôle nlus adéquat.

Les travaux sont d'inégale longueur et d'inégale valeur. Ils sont tous suivis par un résumé des discussions qui, sur le plan pratique, sont d'un réel intérêt, et parfois d'une portée appréciable. Quelques-uns s'imposent tant par leur actualité que par leur justesse: «Employee Motivation », par R.L. Kahn; "The Shorter Workweek Issue », par W. Harber et F.W. Climer; «Employee Benefits - Their Current Patterns and Trends », par J.V. Strong; «Supplemental Unemployment Benefits: Principles and Problems », par R.A. Leutheuser.

«Addresses on Industrial Relations》 est recommandé pour ceux qui sont engagés dans la direction du personnel. Ils y trouveront une source de pensée capable d'orienter leur action future.

\section{L.-M .TrEMBLAY}

